\title{
A Biologically-Inspired Sensor Wakeup Control Method for Wireless Sensor Networks
}

\author{
Yan Liang ${ }^{\mathrm{a} 1}$, Member, IEEE, Jiannong Cao ${ }^{\mathrm{b}}$, Senior Member, IEEE \\ Lei Zhang ${ }^{\mathrm{b}}$, Member, IEEE, Rui Wang ${ }^{\mathrm{c}}$, and Quan Pan ${ }^{\mathrm{a}}$, Member, IEEE \\ ${ }^{a}$ College of Automation, Northwestern Polytechnical University, Xi'an, China \\ ${ }^{\mathrm{b}}$ Department of Computing, The Hong Kong Polytechnic University, Hong Kong \\ ${ }^{\mathrm{c}}$ Institute of Computing Technology, Chinese Academy of Sciences, Beijing, China
}

\begin{abstract}
This paper presents an artificial ant-colony approach to distributed sensor wakeup control in wireless sensor networks (WSN) to accomplish the joint task of surveillance and target tracking. Each sensor node is modeled as an ant and the problem of target detection is modeled as the food locating by ants. Once the food is found, the ant will release pheromone. The communication, invalidation, and fusion of target information are modeled as the processes of pheromone diffusion, loss and accumulation. Since the accumulated pheromone can measure the existence of a target, it is used to determine the probability of ant searching activity in the next round. To the best of our knowledge, this is the first biologically-inspired sensor wakeup control method in WSN. Such a biologically-inspired method has multiple desirable advantages. First, it is distributive and does not require a centralized control or cluster leaders. Therefore, it is free of the problems caused by leader failures and can save the communication cost for leader selection. Second, it is robust to false alarms because the pheromone is accumulated temporally and spatially and thus is more reliable for wakeup control. Third, the proposed method does not need the knowledge of node position. Two theorems are presented to analytically determine the key parameters in the method: the minimum and maximum pheromone. Simulations are carried out to evaluate the performance of the proposed method in comparison with representative methods.
\end{abstract}

Keywords: Sleep/Wakeup Control, Wireless Sensor Networks, Surveillance, Target Tracking, Ant Colony

\footnotetext{
1 This research is supported by the Hong Kong RGC General Research Grant (PolyU 5102/07E), the National Science Foundation Council of China (no. 60634030), the Scientific and Technological Innovation Foundation of NPU, the Program for New Century Excellent Talents of University, the National Basic Research Program of China (no. 2006CB303000), and the Beijing Natural Science Foundation (no. 4092045).

Corresponding authors: Jiannong Cao (csjcao@comp.polyu.edu.hk), Lei Zhang (cslzhang@comp.polyu.edu.hk). 


\section{INTRODUCTION}

With the rapid development of micro-electro-mechanic and wireless communication techniques, it becomes feasible to deploy a large-scale wireless sensor network (WSN) with thousands of tiny and inexpensive sensor nodes, which are scattered over a vast field for the sensing tasks [1]. One of the common applications of WSN is to detect and track the target, such as an intruder toward forbidden zones or a tank in the battlefield [2]. WSN has great potentials in military and civil applications, including battlefield surveillance, environmental protection and traffic monitoring [3][4].

For each sensor node, the probability of target occurrence is statistically low because the sensing vicinity of a single node occupies only a very small portion of the whole sensing zone. Therefore, sensing without wakeup control will spend much energy in the absence of target. Even a target appears, sensing without wakeup control will still consume too much energy for processing and transmitting excessively redundant information due to the dense node deployment. In many applications, the sensors are driven by un-rechargeable micro-batteries with very limited power. Therefore it is of high demand to develop effective sensor wakeup control (SWC) schemes for energy-efficient sensing tasks. In general, SWC can be classified into three categories: surveillance-oriented, tracking-oriented, and topology-oriented [5]-[15].

In surveillance, it is unknown when and where a target will appear. Thus, the sensor nodes are required to work in turns to achieve a homogeneous coverage of a wide area with even energy consumption [7]. In the surveillance-oriented SWC, the sensor nodes switch their operation modes between "sleep" and "wakeup" independently and randomly with a preset probability. The surveillance-oriented SWC can be implemented by Random Independent Sleeping (RIS) [8] asynchronously or Randomized Activation (RA) [9] synchronously. The surveillance-oriented SWC is simple to implement. It is free of the communication cost for node collaboration and does not need node localization. However, due to the lack of node collaboration, it cannot use the target information collected in a neighborhood to improve the wakeup control efficiency.

In target tracking, the target moves and even maneuvers. The tracking-oriented SWC focuses on how to dynamically wake up the nodes surrounding the moving target in order for intensive node coverage of the target [5]. The tracking-oriented SWC uses one or more cluster leaders, which are in charge of collecting the target reports, predicting the target position and waking up the nodes in the vicinity of the predicted target location. In the Selective Activation (SA) scheme [9], the leader wakes up the nodes within the circle 
centered at the predicted target with radius being the node sensing range. The awaken nodes try to detect the target, and go back to "sleep" if no target is found. The target is located as the centroid of the nodes that discover the target. The location of the target at the next time is predicted as the linear combination of the two latest estimates of target location. In the Dynamic Convoy Tree-based Collaboration (DCTC) scheme [10], the nodes that detect the target will cooperatively select a root and construct an initial tree. The root collects information from the tree nodes, predicts the target movement, and chooses the tree nodes for power-efficient tracking. However, in DCTC it is assumed that the target exists and has been detected. Moreover, DCTC requires accurate node localization. A big node localization error will lead to a significant prediction offset of target position and further deteriorate the performance of wakeup control.

The third strategy of SWC, such as the PEAS-based (PEAS: Probing Environment and Adaptive Sleeping) [8] scheme, was originated from the study of network topology control [11]-[15] and can be used for both surveillance and target tracking. The surveillance zone is divided into many clusters. The sensor nodes in the same cluster periodically elect their leader to gather the target information. The cluster members randomly switch between "Wakeup" and "Sleep". If the leader finds a target, it will wakeup all cluster members, while if a member finds a target, it will wakeup its neighbors. As a direct combination of surveillance-oriented and tracking-oriented SWC, however, the topology-oriented SWC suffers from false alarms because any target-existence reported by a single node will wake all its neighbors. The false alarms will lead to severe false wakeups, especially for node-intensive WSN. Nonetheless, false alarms are inevitable under complex environments. Thus it is highly demanded to develop new SWC methods which are robust to false alarms in joint surveillance and tracking.

This paper presents an artificial ant colony based approach to online distributed sensor wakeup control. To the best of our knowledge, this is the first biologically-inspired sensor wakeup control method for WSN. The proposed method has many desirable properties. First, it is a joint surveillance and tracking scheme. The positive-feedback accumulation of pheromone supports the prompt task switches between surveillance and tracking. Second, the pheromone accumulation integrates the temporal and spatial information of a target so that it is robust to false alarms. Third, the proposed scheme requires neither a centralized control nor cluster leaders. Thus many problems caused by leader failures are avoided and the communication cost of leader selection is saved. Moreover, it does not need to know the node position and thus is free of node localization.

The rest of the paper is organized as follows. Section II presents the ant-colony method for sensor 
wakeup control. Section III presents two theorems for parameter determination according to the sensing requirements. Section IV presents simulations to evaluate and analyze the proposed method. Section V concludes the paper.

\section{The Biologically-InSPIREd Scheme for Sensor WaKeUp Control}

In some biological collective systems, e.g. ant colonies, each individual has very limited ability of sensing, processing, memorizing and communicating, and hence has little capability to accomplish global objectives alone. However, via seemingly simple interaction among multiple individuals, the global objectives can be reached due to the emergence behavior [16][17], [29]-[32]. In recent years, much attention has been paid to distributive optimization with collective intelligence, especially ant colony optimization [18]-[22], [33]-[36]. The biologically-inspired modeling has many merits. For example, each individual only needs to do simple response without complex computing, memorizing or communicating, and hence the energy cost for each node is very limited. Swarm intelligence, such as artificial ant colony, has positive feedback mechanism, which supports prompt task switches. The optimization can be accomplished even in the case that each individual is much limited in sensing ability. Recently, many works have been done on the biologically-inspired WSN modeling, such as cooperative routing [23], operating system design [24], thread management [25], time synchronicity [26] and sensor movement strategy [27]. However, little research has been reported on the biologically-inspired SWC. To this end, in this paper we propose a biologically-inspired SWC scheme for joint surveillance and tracking.

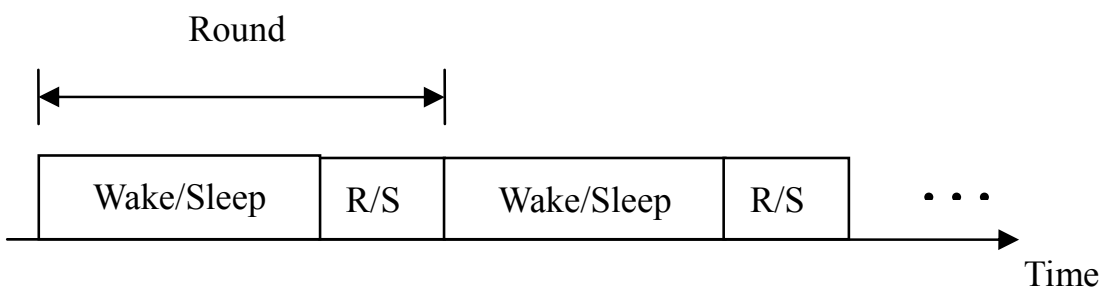

Figure 1. Operation flow map of the Round based wake/sleeping protocol.

In WSNs, the clock in each node needs periodical time synchronization for node collaboration. As in many other works such as RA [9], SA [9], DCTC [10] and PEAS-based [12] methods, in this paper we assume that the time synchronization is guaranteed, and we consider a class of commonly-used time 
synchronization protocols, the Round-based synchronization protocols (such as the S-MAC protocol [11]) as shown in Fig. 1. In such protocols, each operating period (called Round) has two phases, "Wake/Sleep" and "R/S". In the phase of "Wake/Sleep", a node randomly chooses the state of "Wake" or "Sleep" according to its "Wake Probability". The node in the "Wake" state turns on its sensing module and initializes target detecting, while the node in the "Sleep" state turns off its sensing module for energy saving. In the phase of " $\mathrm{R} / \mathrm{S}$ ", the nodes receive messages for collaboration and only the nodes that find the target in this Round will send messages of target information to their neighbors. Besides sending messages in the " $\mathrm{R} / \mathrm{S}$ " phase, each node will also periodically send to its neighbors the message stamped by its local time clock for time synchronization. Such time synchronization is independent of application-level wakeup control and hence is not considered in designing the wakeup control strategy.

Consider the homogeneous binary WSN [9] that is composed of immobile sensor nodes with the same configuration, such as the same sensing range, the same communication range, etc. In each $\mathrm{R} / \mathrm{S}$, the sensor node in "Wake" state outputs "1", representing "Target Existence" with detection probability $P_{d}$ in the presence of the target within its sensing range, or with false alarm probability $P_{f}$ in the absence of the target within its sensing range. Otherwise it will output " 0 ", representing "No Target". Although such a basic configuration is very simple, it is very challenging because there are no super nodes to support the computationally intensive central processing and control, no mobile nodes to provide additional assistances, and no powerful sensors to provide abundant information of target position and attributes.

The goal of this paper is to control the wakeup state of each node in each Round in order to accomplish the task of surveillance and tracking in an energy-efficient and distributive style. Here "wakeup" refers to "wake up sensing module" because the communication module only works in the "R/S" phase.

Remark 1: It is worth mentioning that it is possible to separately control the sensing module and the communication module of each node. For node collaboration, the Round-based synchronization protocol is considered in our problem formulation. This protocol is very effective in saving communication costs due to the fact that the communication module does not work in the phase of "wake/sleep" and works in the phase of "R/S" (among the states of transmitting, receiving and idle listening), while the "wake/sleep" phase is much longer than the "R/S" phase in a Round. In other words, the energy consumption of the communication module has already been effectively saved by the Round-based synchronization protocol. Thus this paper focuses on controlling the sensing modules in a distributive style. 
Remark 2: One may doubt about the importance of optimization on sensor wakeup control by the fact that the sensing module consumes much less energy than the communication module, given the same operation time interval. In our opinion, controlling sensor wakeup is necessary with the following reasons:

- The communication module sleeps in the "wake/sleep" phase, which takes up most of the portion in each Round. Hence the permitted communication operation time interval is significantly smaller than the permitted sensing operation time interval.

- The proposed sensor wakeup control does not only save the energy consumption of the sensing module. If a waked sensor finds a target, then additional energy-consumption activities, including data storage, processing and even transmission will be further triggered, which will increase much the energy consumption.

Therefore, the wakeup control method is expected to reduce the redundant target detection or the resultant redundant message transmission, and hence reduce the energy consumption on communication.

If there are $n$ binary sensor nodes, then the optimal control scheme would be selected from $2^{n}$ possible choices in each Round. Obviously, this is a combinatorial optimization problem, which motivates our investigation on ant colony optimization (ACO) to find a solution. The ACO method proposed by Dorigo et al [18] is a new paradigm for stochastic combinatorial optimization. ACO is inspired by the foraging behavior of ants. When searching for food, ants explore the area surrounding their nest in a random manner. Once an ant finds food, it comes back to the nest. During the return trip, the ant will release a kind of chemical pheromone on the trail. The released pheromone will guide other ants to find the food. Finally, the shortest path between the ant nest and the food can be established. The ACO based algorithms have been successfully used in many applications [19]-[21]. Although having many variants, the ACO methods have some common characteristics: the use of a natural metaphor, inherent parallelism, stochastic nature, adaptivity, and the use of positive feedback [19]. As mentioned in [19], a method meeting all these characteristics can be viewed as a member of biologically-inspired methods.

In our model, each sensor node is viewed as an ant, while the target is viewed as the moving food to be found. The target detection activity of a node is modeled as the food search process of an ant as follows. The event that an ant randomly walks to search the food within its search zone represents the random target detection by a node within its sensing zone; the search zone of an ant is the sensing zone of that node, i.e. the discs area centered at the node with the diameter being the sensing range; the probability that an ant finds the 
food within its search zone equals to the detection probability $P_{d}$, i.e. the probability that the node detects the target within its sensing range; the probability that an ant falsely confirms the food existence in the absence of the food within its search zone equals to the false-alarm probability $P_{f}$, i.e. the probability that the node falsely confirms the existence of the target.

By establishing the corresponding relationship between the node detection and ant search, the SWC problem is transformed into the distributive on-off control of the food search activity of each ant. As illustrated in Fig. 2, we propose a novel SWC scheme based on the following collaboration processes of ant colony: “Ant Search", "Pheromone Release", "Pheromone Accumulation" and "Pheromone based Control”.

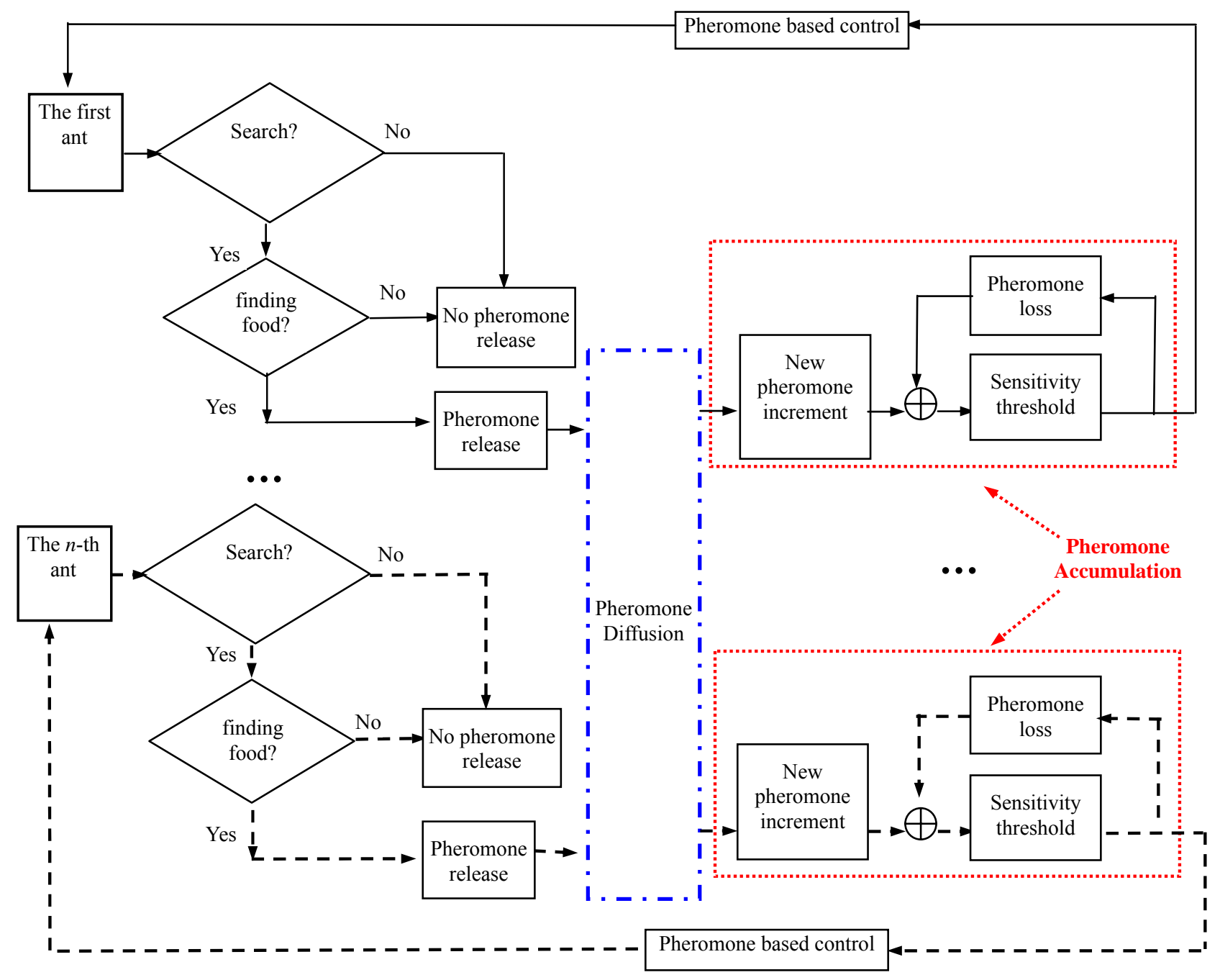

Figure 2. Operation flow chart of the ant-colony control method.

In the "Ant Search" process, an ant performs random search or not according to its search probability. If an ant is in the "Search" state, it will randomly walk within its search zone for possible food. Otherwise, it will not move. In the "Pheromone Release" process, the ant will release pheromone if it finds the target. In 
the "Pheromone Diffusion" process, the newly-released pheromone diffuses and then evenly distributes within the vicinity of the ant. Such a pheromone diffusion process imitates the spread of target information via node communication. In the "Pheromone Accumulation" process, the pheromone is accumulated from two sources, the newly-released pheromone and the previously-released pheromone. In the "Pheromone based Control" process, the pheromone, as the measure of the possibility of food existence, is used to determine the search probability of each ant in the next Round. The algorithm is described as follows.

Ant Search. The indicator of the state of an ant is defined as

$$
S_{j}(k)= \begin{cases}1 & \text { ant } A_{j} \text { is on search duty in the } k \text {-th Round } \\ 0 & \text { otherwise }\end{cases}
$$

where $A_{j}$ represents the $j$-th ant. In the $k$-th Round, the event $S_{j}(k)=1$ happens with the "Search Probability" $w_{j}(k)$, which is determined in the (k-1)-th Round. The "Ant Search" process can be described by using the following pseudo-code.

\{

for $j=1: n \quad / / n$ is the total number of sensor nodes

Generate an independent random sample, $s_{j}(k)$, using the uniform distribution within $[0,1]$.

$$
\begin{aligned}
& \text { if } s_{j}(k) \leq w_{j}(k) \text {, then } S_{j}(k)=1 \\
& \text { else } S_{j}(k)=0 \\
& \text { end }
\end{aligned}
$$

end

\}

If an ant is not on search duty, it will wait until the next Round so that the energy is saved for future use. Otherwise the ant randomly walks within its search zone to search for the possible food.

Pheromone Release. A searching ant will confirm the food existence in two cases. The first case is that the food exists and the ant finds it. This case is characterized by the node detection probability $P_{D}$. The second case is that the food does not exist but the ant makes a false confirmation. Such case is characterized by the node false alarm probability $P_{f}$. Once the food existence is confirmed, the ant will release a unit pheromone 
increment and stop searching in this Round. The indictor of the food existence is defined as

$$
E_{j}(k)=\left\{\begin{array}{cc}
1 & \text { The food exists in the search zone of } A_{j} \text { in the } k \text {-th Round } \\
0 & \text { otherwise }
\end{array}\right.
$$

and the indictor of food existence confirmation is defined as

$$
D_{j}(k)=\left\{\begin{array}{cc}
1 & A_{j} \text { confirms that the food exists in the } k \text {-th Round } \\
0 & \text { otherwise }
\end{array}\right.
$$

If the $j$-th ant confirms that the food exists, then it will release a unit pheromone increment:

$$
\tau_{j}(k)=\left\{\begin{array}{cc}
1 & D_{j}(k)=1 \\
0 & \text { otherwise }
\end{array} \quad \forall j=1, \cdots, n\right.
$$

Releasing pheromone means that the node releases the target existence information to its neighbors. "Pheromone Release" models the activity of node detection and can be implemented as follows.

\{

if $S_{j}(k)=1$ and $E_{j}(k)=1$, then

Generate an independent random sample, $d_{j}(k)$, using the uniform distribution within $[0,1]$.

if $d_{j}(k) \leq P_{d}$, then $D_{j}(k)=1$ and $\tau_{j}(k)=1$; end

else if $S_{j}(k)=1$ and $E_{j}(k)=0$, then

Generate an independent random sample, $f_{j}(k)$, using the uniform distribution within $[0,1]$.

if $f_{j}(k) \leq P_{f}$, then $D_{j}(k)=1$ and $\tau_{j}(k)=1$; end

else

$D_{j}(k)=0$ and $\tau_{j}(k)=0$.

end

\}

Pheromone Diffusion. Denote by vicinity $(j)$ the ant collection in the vicinity of the $j$-th ant:

$$
\operatorname{vicinity}(j)=\left\{i \mid i \in\left\|l_{i}-l_{j}\right\| \leq R_{c}\right\} \quad j=1, \cdots, n
$$

where $l_{k}$ is the spatial location of the $k$-th ant. The localized collaboration range $R_{c}$ is the communication radius. Denote by $|\operatorname{vicinity}(j)|$ the amount of the ants within vicinity $(j)$. Since a node will exchange message about its local time clock with its neighbors periodically, it knows the list of neighbors and thus 
$|\operatorname{vicinity}(j)|$ is known. It is worth to note that $|\operatorname{vicinity}(j)|$ is dynamically updated so that it is adaptive to the changes of $\mid$ vicinity $(j) \mid$, e.g. the changes due to node failures.

The newly released pheromone of ant $A_{j}$ diffuses from its discs area to the neighboring discs areas and finally distributes uniformly within these discs areas of vicinity $(j)$. The concentration of newly released pheromone of ant $A_{j}$ is $\tau_{j}(k) /|\operatorname{vicinity}(j)|$. Pheromone diffusion depicts the spread of food information.

Pheromone Accumulation. In the discs area where $A_{i}$ locates, the pheromone concentration is uniform and comes from two sources. One is the newly-released pheromone increment $\Delta I_{i}(k)$ of $A_{i}$ and/or its neighbors

$$
\Delta I_{i}(k)=\sum_{j \in v i c i n i t y(i)}\left(\tau_{j}(k) /|\operatorname{vicinity}(j)|\right)
$$

Another source is the residual of previously-released pheromone. The previously-released pheromone concentration $I_{i}(k-1)$ decreases over time, and in the $k$-th Round the residual pheromone is $(1-\rho) I_{i}(k-1)$, where $\rho \in[0,1]$ is the loss ratio.

As explained in the Max-Min Ant Colony System (MMACS) [20], the pheromone should be bounded. Thus the final accumulated pheromone concentration is

$$
I_{i}(k)=\min \left\{I_{i}^{\max }, \max \left\{I_{i}^{\min },(1-\rho) I_{i}(k-1)+\Delta I_{i}(k)\right\}\right\}
$$

where $I_{i}^{\min }$ and $I_{i}^{\max }$ are the minimum and maximum pheromone levels within the search zone of $A_{i}$. The analytical design of $I_{i}^{\min }$ and $I_{i}^{\max }$ will be discussed in Section III. The initial $I_{i}(0)$ is chosen to be its minimum value $I_{i}^{\min }$.

Pheromone-based Control. A higher pheromone concentration implies a higher probability of food existence. Therefore the search probability should increase monotonically with the pheromone concentration because the ant closer to the food has higher possibility to find the food and thus should have more search opportunity. The pheromone concentration is linearly mapped to the "Search Probability" as follows:

$$
w_{i}(k+1)=I_{i}(k) / I_{i}^{\max } \quad i=1, \cdots, n
$$

With the search probability defined in (2.8), an ant randomly determines whether or not it should search the 
food in the $(k+1)$-th round. $w_{i}(0)$ is initialized to be its minimum value $I_{i}^{\min } / I_{i}^{\max }$.

It should be noted that the proposed SWC method is not a standard ACO approach. First, the optimal solution in the standard ACO is the shortest path, which is static and deterministic because the food is immobile. However, the optimal solution in the proposed method is temporally dynamic and stochastic because the food moves randomly. Second, in our method the random movement of an ant is limited within its local search zone, instead of the whole search space as in the standard ACO. Third, in our method the pheromone determines whether or not an ant makes a random search, instead of which search path should be chosen as in the standard ACO.

The proposed SWC scheme has multiple interesting properties. First, the implementation is distributive without requiring any leader. Therefore it has high robustness to node and communication failures. Meanwhile, no additional communication expense of leader selection is needed. Second, it does not require the information of node position. Third, the positive feedback mechanism in collaborative optimization of ant colony supports fast state switches between "wake" and "sleep". Fourth, the pheromone for wakeup control is accumulated temporally and spatially so that the wakeup control is robust to false alarms.

\section{Parameter Design}

As indicated in [27], a proper selection of minimum and maximum pheromone levels can significantly improve the target search ability of ant colony. However, these two important parameters were determined $a$ priori in MMACS [27]. In this section, we will present two theorems to design the two parameters $I_{i}^{\min }$ and $I_{i}^{\max }$ analytically according to the sensing task.

Consider the case that the target appears at a random time and at a random location, and then moves randomly within a random length of time before disappearing [8]. This is the most difficult case of target sensing because there is no rule of target movement. The performance indexes in such case can be viewed as the most conservative measure of sensing ability. The target location can be modeled by the independently uniform distribution within the surveillance zone. We have the following two theorems.

Theorem 1: Consider the homogeneous binary WSN where sensor nodes have the same configuration. The nodes are immobile after deployment and output binary confirmation: "Target Existence" or "No Target". Suppose that the target location follows the independently uniform distribution within the surveillance zone. 
It is confirmed with the confidence degree $\beta$ that

(a)

$$
|\Psi(k)| \leq M_{\max }
$$

where $M_{\max }$ satisfies

$$
p m f_{1}\left(M_{\max }-1\right)<\beta \leq p m f_{1}\left(M_{\max }\right)
$$

with

(b)

$$
\begin{gathered}
\operatorname{pmf}_{1}(M) \underline{\Delta} P\{|\operatorname{vicinity}(j)| \leq M\}=\sum_{r=0}^{M} \frac{e^{-\lambda_{1}} \lambda_{1}^{r}}{r !} \\
\lambda_{1}=n \pi R_{c}{ }^{2} /\|\Omega\|
\end{gathered}
$$

In (3.1) (3.5), $\Psi(k)$ is the collection of sensor nodes within the circle $R(k)(R(k)$ centers at the location of the target and its radius is set as the sensing distance $s) ;|\Psi(k)|$ is the node number of $\Psi(k)$; $|\operatorname{vicinity}(j)|$ is the number of sensor nodes within vicinity $(j)$ defined in $(2.5)$; $n$ is the total number of sensor nodes within the surveillance zone; $R_{c}$ is the localized collaboration distance in (2.5); $\|\Omega\|$ is the area of total surveillance zone $\Omega ; P_{D}$ is the detection probability of each sensor node; $P_{f}$ is the false-alarm probability of each sensor node; and $\rho$ is the loss ratio.

Proof: See Appendices A and B.

As shown in Theorem 1, the maximum pheromone level $I_{j}^{\max }$ is a function of variables from three categories. The first category includes the parameters of WSN, such as the false-alarm probability, detection probability, sensing distance and the number of sensor nodes. The second category includes the environment parameter - the area of total surveillance zone. The last category includes the parameters of ant-colony method, such as the localized collaboration distance and the pheromone loss ratio.

Theorem 2: Consider the homogeneous binary WSN where sensor nodes have the same configuration. The nodes are immobile after deployment and output binary confirmation: "Target Existence" or "No Target". If there exists a target in the surveillance zone in the $k$-th Round, then it is confirmed with the confidence degree $1-\alpha$ that

(a)

$$
|\Psi(k)| \geq M_{\text {min }}
$$


where $M_{\min }$ satisfies

$$
p m f_{2}\left(M_{\min }\right) \leq \alpha<p m f_{2}\left(M_{\min }+1\right)
$$

with

(b)

$$
\begin{aligned}
& p m f_{2}(M) \underline{\underline{\Delta}} P\{|\Psi(t)| \leq M\}=\sum_{r=0}^{M} \frac{e^{-\lambda_{2}} \lambda_{2}^{r}}{r !} \\
& \lambda_{2}=n \pi s^{2} /\|\Omega\|
\end{aligned}
$$

In (3.6) (3.10), $\Psi(k)$ is the collection of sensor nodes within the circle $R(k)(R(k)$ centers at the location of the target and its radius is set as the sensing distance $s) ;|\Psi(k)|$ is the node number of $\Psi(k)$; $|\operatorname{vicinity}(j)|$ is the number of sensor nodes within vicinity $(j)$ defined in $(2.5) ; n$ is the total number of nodes in surveillance zone; $R_{c}$ is the localized collaboration distance in (2.5); $\|\Omega\|$ is the area of total surveillance zone $\Omega ; P_{D}$ is the detection probability of each sensor node; $P_{f}$ is the false-alarm probability of each sensor node; $\rho$ is the pheromone loss ratio; $P_{\min }$ is the minimum surveillance probability; and $M_{\max }$ is determined by (3.2)-(3.4).

Proof: See Appendices A and C.

As shown in Theorem 2, the minimum pheromone level is a function of variables from four categories. The first category includes the parameters of WSN, such as the false-alarm probability, detection probability, sensing distance and the number of sensor nodes. The second category includes the environment parameterthe area of total surveillance zone. The third category includes the performance index - the minimum surveillance probability. The last category includes the parameter of ant-colony method - localized collaboration distance.

\section{SimULATIONS}

Similar to [8][28], we consider a binary WSN with 500 sensors randomly and uniformly deployed on a planar area of $200 \mathrm{~m} \times 200 \mathrm{~m}$. All sensor nodes are identical and have the same configuration: $R_{c}=30 \mathrm{~m}$, $s=15 \mathrm{~m}, P_{d}=0.9, P_{f}=0.05$ and $P_{\min }=0.3$. The target goes into the field at a random location and then moves 
in Brownian motion. The Brownian motion is driven by a zero-mean Gaussian noise process with variance $400 \mathrm{~m}^{2}$ in consecutive 10 Rounds. The target appears at time 20 and disappears at time 120.

In the proposed ACO-like method, the maximum and minimum pheromones are designed by using the presented two Theorems. We set $\rho=0.4$ and $I_{i}(0)=I_{i}^{\min }$. Two state-of-the-art SWC strategies introduced in the Introduction Section are used for comparison. One is the topology-oriented PEAS-based scheme with the same parameter configuration as in [8]. The leaders are selected using the standard PEAS method [12] at the beginning. Another is the surveillance-oriented RIS [9] strategy. We consider three RIS schemes: "RIS-high probability", "RIS-middle probability" and "RIS-low probability" with wakeup probability being 1, 0.5 and 0.077, respectively. The "RIS-high probability" represents the case that all nodes remain awake and thus reaches the upper bound of sensing ability with maximum energy cost. The "RIS-low probability" (i.e. the wakeup probability is the lowest wakeup probability of the proposed artificial ant-colony approach) represents the lower bound of sensing ability with minimum energy cost. The "RIS-middle probability" is the tradeoff between "RIS-high probability" and "RIS-low probability". The tracking-oriented strategy is not considered in the comparison because it requires node localization.

Fig. 3 plots the curves of the total number of waked sensor nodes versus time. As shown in Fig.3, the three RIS schemes have a constant number of waked sensor nodes in the process of surveillance and target tracking. This is because its wakeup strategy cannot tell surveillance from tracking due to the lack of node collaboration. It can be seen that the PEAS based scheme has 100 leaders after initialization. Though there is no target in the interval $[0,20]$, the number of waked sensor nodes in the PEAS based scheme still significantly increases to more than 400 because of the sensor false alarms.

In time intervals $[0,20]$ and $[120,140]$, where there is no target, the proposed scheme approaches to the "RIS-low probability" as shown in Fig.3. This validates the effectiveness of the presented parameter design Theorems. In interval [20, 120], where the target appears, the total number of waked sensor nodes increases. This validates that the proposed wakeup control scheme is adaptive to the task switches between surveillance and target tracking. The number of waked sensor nodes by the proposed method is much smaller than that by the PEAS based scheme because the proposed scheme exploits both temporal and spatial accumulation of the target information and thus it is robust to false alarms. After time 120, the target leaves the surveillance zone and the number of waked sensor nodes by the proposed scheme promptly returns to the minimum level. It implies that the positive feedback mechanism of pheromone accumulation supports the fast task switch 
between surveillance and target tracking.

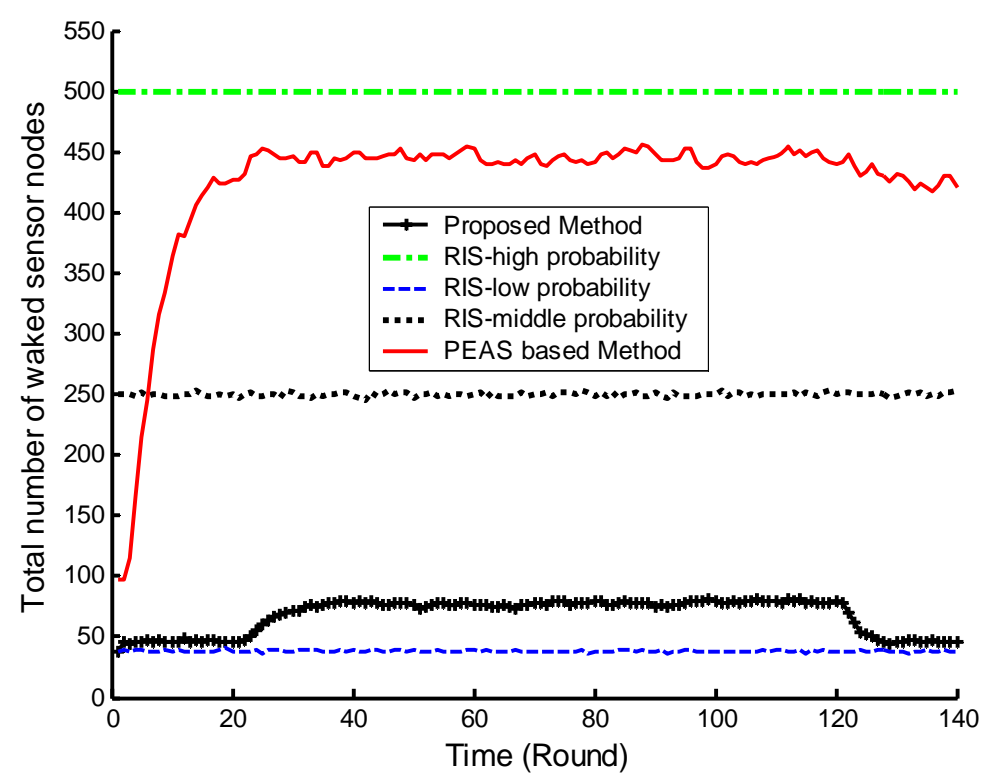

Figure 3. Total number of waked sensor nodes by different schemes.

Fig. 4 shows the number of valid waked sensor nodes that detect the true target. The curves of PEAS based scheme and the "RIS-high probability" scheme are almost overlapped, which implies that they wake up almost all the valid nodes. This is because the PEAS based scheme wakes up all possible valid sensor nodes for the best sensing ability. However, as shown in Fig. 5, the PEAS based scheme and the three RIS schemes have almost the same ratio of the number of valid waked sensor nodes to that of the total waked sensor nodes, which is much smaller than that of the proposed method.

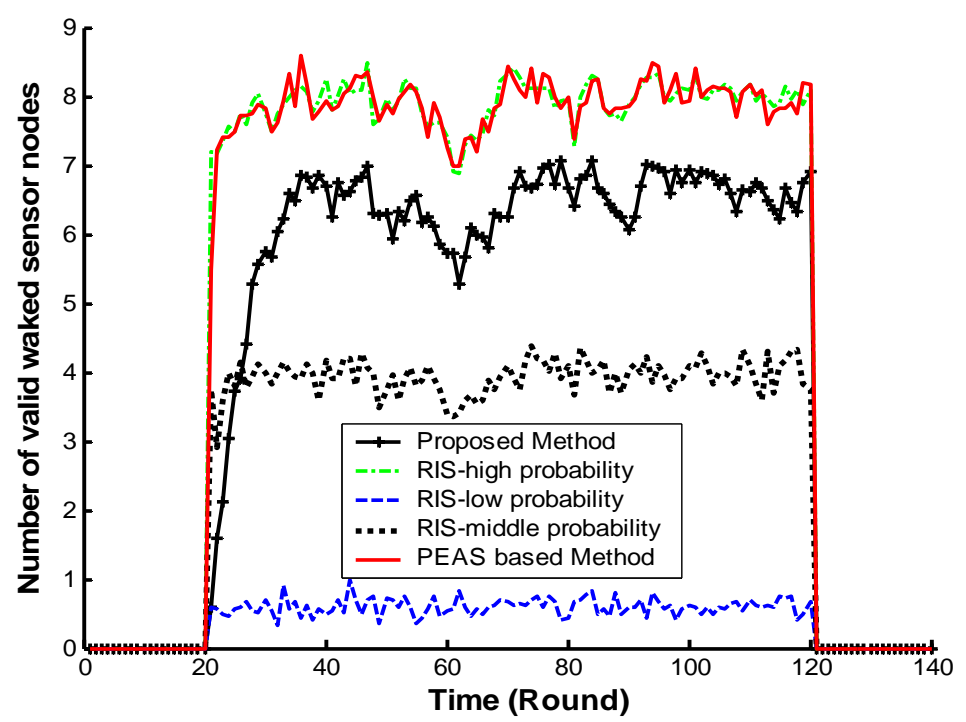

Figure 4. Number of valid waked sensor nodes by different schemes. 


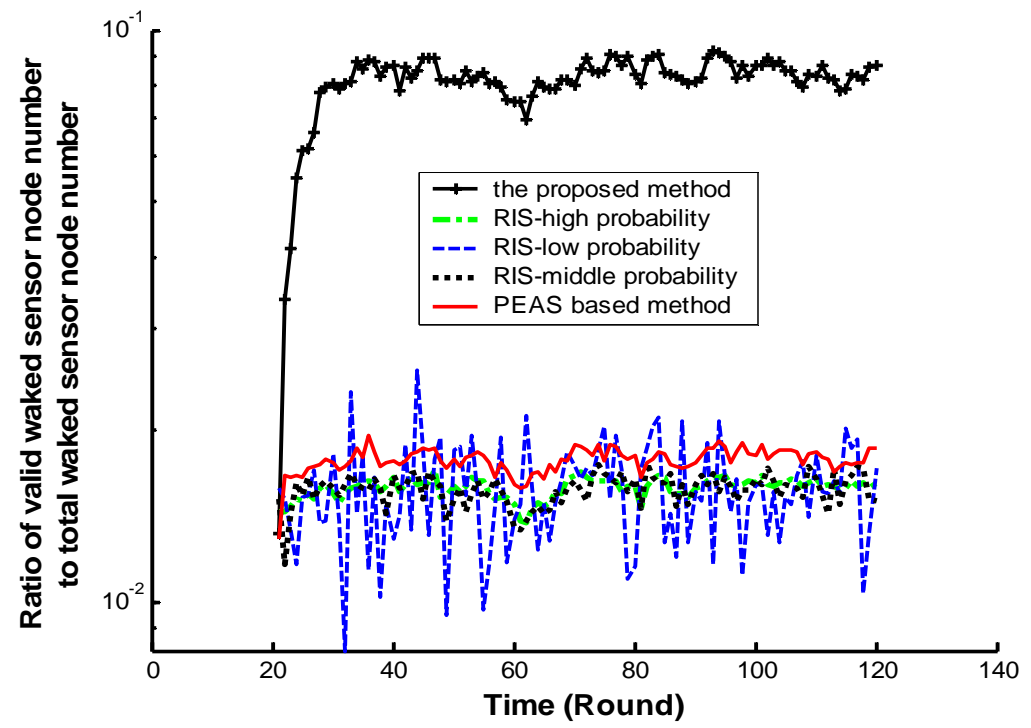

Figure 5. Ratio of the number of valid waked sensor nodes to the number of total waked sensor nodes.

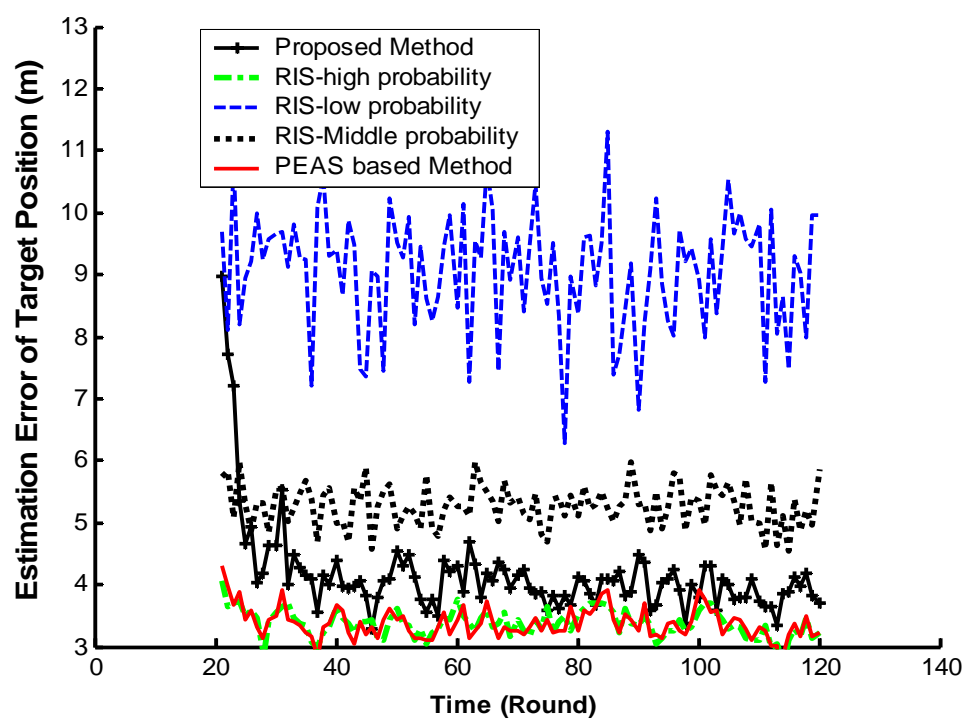

Figure 6. Estimation error of target position by different schemes.

Fig. 6 shows the estimation error of target position, i.e. the distance between the actual target position and the centroid of the valid waked sensor nodes. Again, it can be seen that the PEAS based scheme and the "RIS-high probability" scheme reach the low bound of positioning error because all valid sensor nodes are waked. From Fig. 3 and Fig. 6, we find that the proposed scheme with about 80 waked sensor nodes approaches to the low bound of position error, which is reached by the RIS scheme with 500 waked sensor nodes or the PEAS based scheme with 450 waked sensor nodes. 


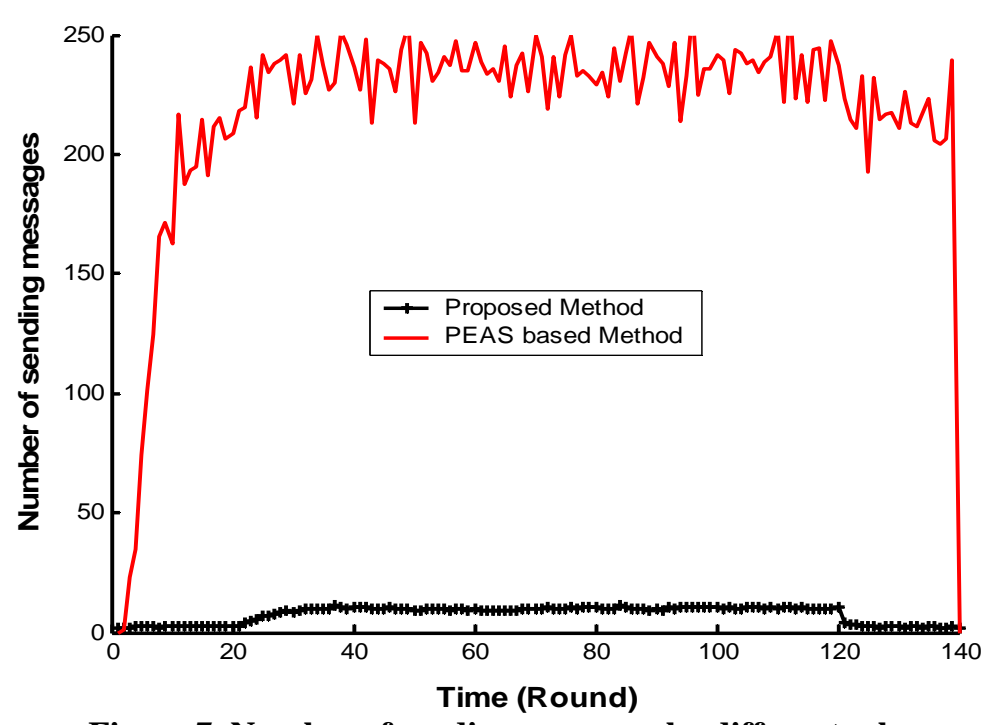

Figure 7. Number of sending messages by different schemes.

The number of sending messages is a key performance index, representing the communication burden caused by collaborative sensing. Fig. 7 shows the statistics of message sending. Because the RIS strategy has no task collaboration, the "RIS-high probability", "RIS-middle probability" and "RIS-low probability" schemes do not need to send any message of the target information for wakeup control. One can see that the proposed method can significantly decrease the number of sending messages compared with the PEAS based scheme because only the nodes that confirm "Target Existence" (no matter it is triggered by the target or false alarm) will send messages in our method.

The PEAS-based scheme is designed to collect the target information as much as possible via node collaboration and thus reaches the upper bounds of the number of valid node and position accuracy. Very differently, the proposed scheme recursively accumulates the temporal and spatial information of the target, which represents the target existence possibility within the vicinity of the node. Such a joint spatial-temporal information accumulation process has high robustness to false alarms and the accumulated target information can reliably and effectively wake up the correct nodes. Therefore, the energy consumption in both sensing and communication is saved.

Apart from the above simulations of tracking a Brownian-motion target, we also performed experiments for the following cases: (1) tracking a constant-velocity (CV) target; and (2) tracking two crossed CV targets as shown in Figs.4-6 and Figs.7-9, respectively. The left column is for the proposed method and the right column is for the PEAS-based method. The RIS method was not used in this experiment because in RIS each node is waked, randomly and independently, with the same wakeup probability so that its wakeup nodes 
remain uniformly scattered. To save space, we only show the wakeup result in a couple of rounds. The results in other rounds are similar. In Figs. 4-9, “*” represents the valid wakeup nodes, which are "Wake” and find the target; "+" represents the invalid wakeup nodes, which are "Wake" but fail to find the target; and "." represents "Sleep" nodes. The target is plotted by " $\square$ " for the proposed method and by " $\square$ " for the PEAS-based method. Through counting the number of "*” and examining whether "*" surrounds the target, we can roughly evaluate the methods' performance to detect and localize the target. Through counting the number of "+", we can evaluate the invalid energy cost. It is clear that the proposed method has a similar number (for example, about 7-9 in the first case) of valid nodes but much less (about 50) invalid nodes compared with the PEAS-based method (with about 120 invalid nodes).
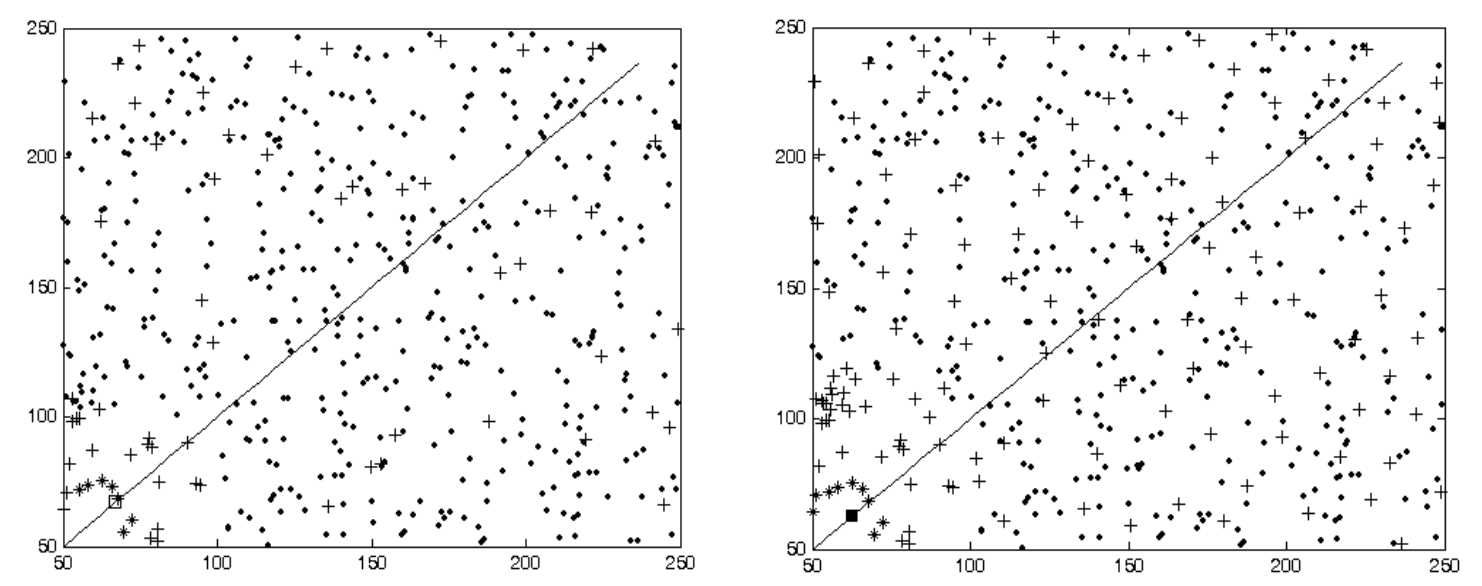

Figure 4. The node state in tracking a CV target in the $8^{\text {th }}$ Round.
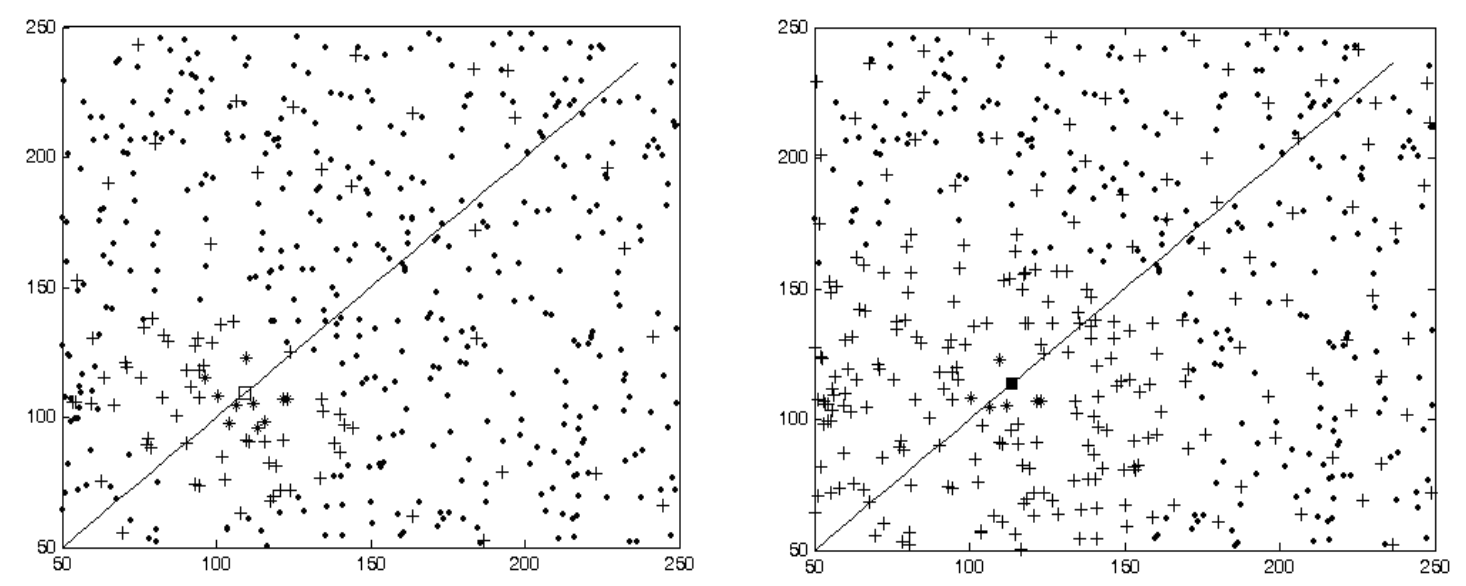

Figure 5. The node state in tracking a CV target in the $14^{\text {th }}$ Round. 

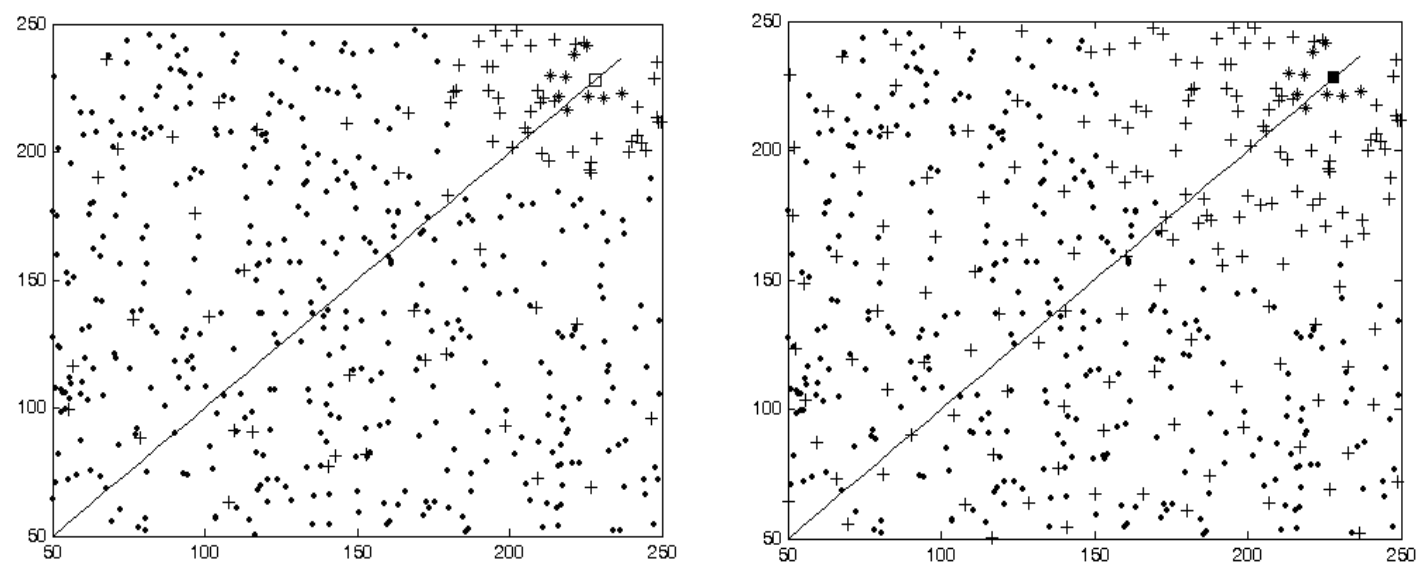

Figure 6. The node state in tracking a CV target in the $39^{\text {th }}$ Round.
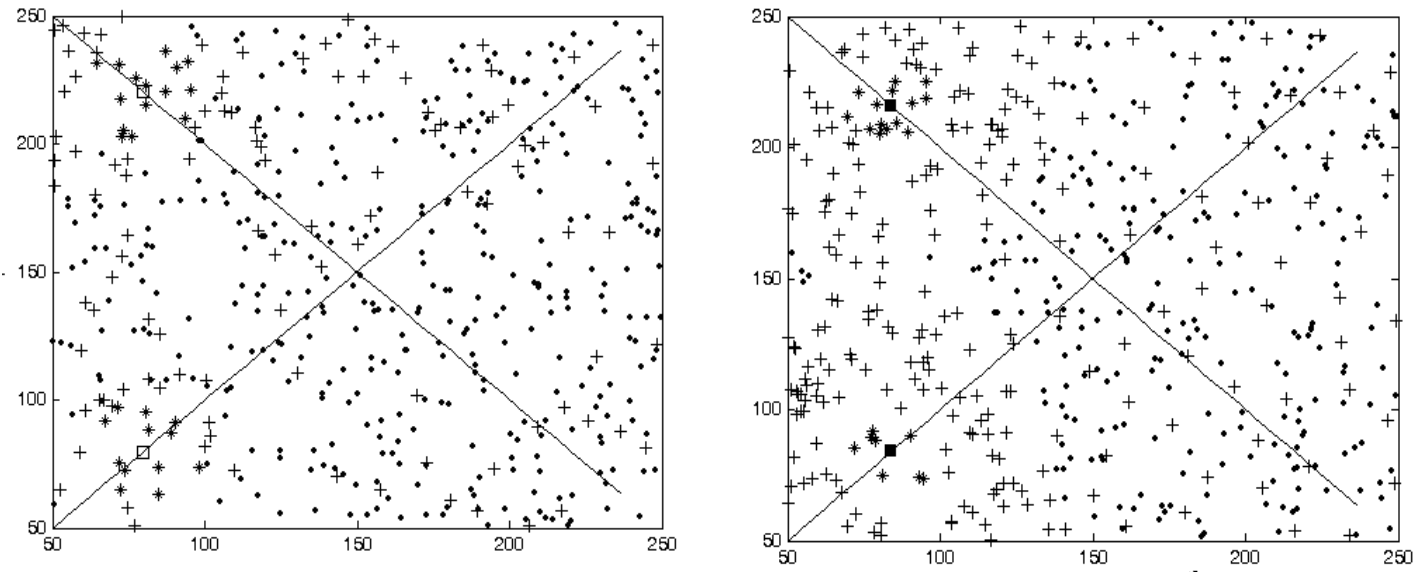

Figure 7. The node state in tracking two crossed CV targets in the $8^{\text {th }}$ Round.
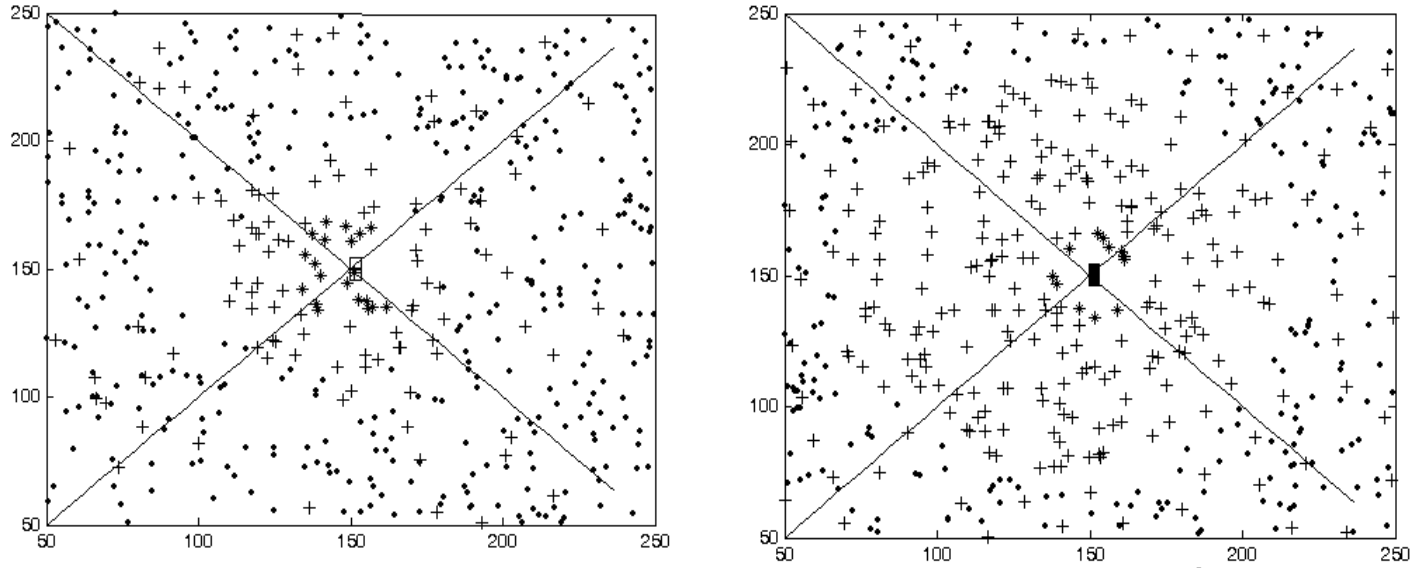

Figure 8. The node state in tracking two crossed CV targets in the $20^{\text {th }}$ Round.
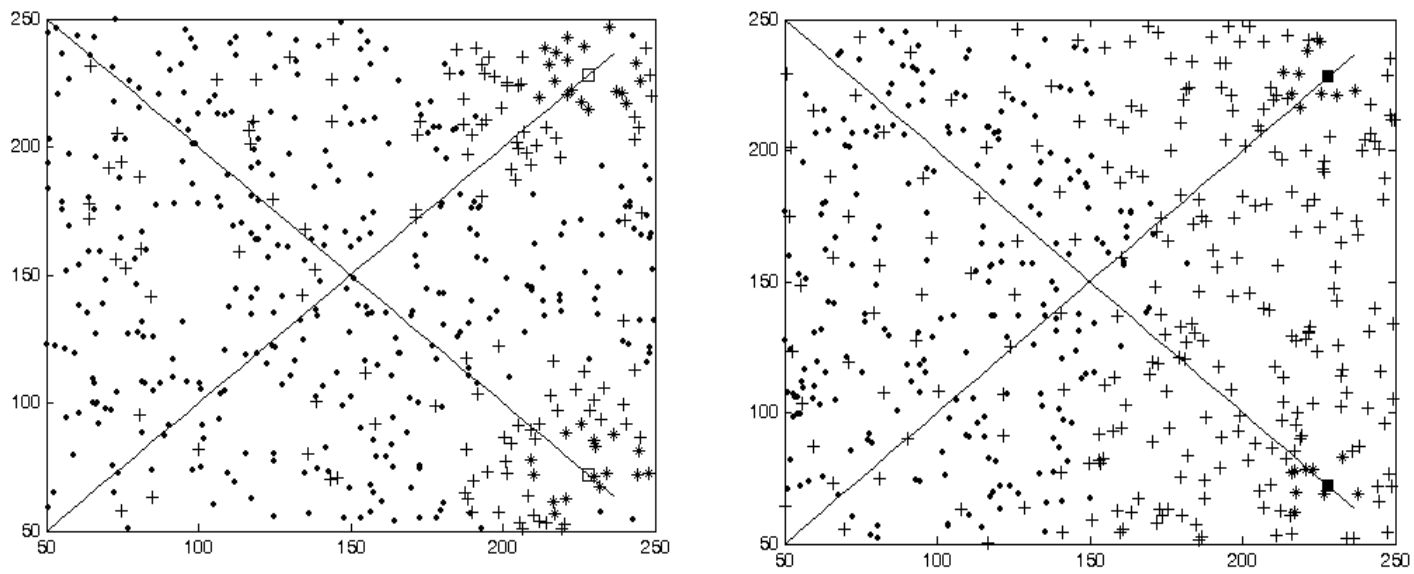

Figure 9. The node state in tracking two crossed CV targets in the $39^{\text {th }}$ Round. 


\section{Conclusion AND Discussion}

This paper presented a biologically-inspired method of sensor wakeup control (SWC) in wireless sensor networks (WSN) for joint surveillance and target tracking. Compared with the existing solutions, the proposed method has many advantages. First, it allows for distributed implementation without requiring a centralized control by cluster leaders. Thus the problems caused by leader failures can be avoided and the additional cost of leader selection is saved. Second, the pheromone is temporally and spatially accumulated for SWC so that the proposed method is robust to false alarms, which are inevitable in WSN due to the complex environment and limited sensing ability of each node. Third, the proposed method does not require node localization. In addition, the minimum and maximum pheromone, which are two key parameters in ant colony-based methods, are analytically designed in the proposed method. Simulations were carried out to demonstrate the efficiency of the proposed algorithm.

One merit of the proposed biologically-inspired SWC is that it does not need the information of node location. However, at the application level, node location is often required. Therefore, one interesting question is that if it is possible to further save the power consumption in the proposed method by introducing a start-up node location procedure? From the viewpoint of information fusion, adding a start-up node location procedure will certainly introduce more information and hence lead to further improvement. However, how to design the corresponding algorithm needs more investigation because utilizing such information will require additional computational and storage cost. To the best of our knowledge, designing such a start-up node location method is still an open problem because the associated biological paradigm is not available yet.

In the proposed method, the adjacent nodes exchange information and node wakeup is involved in data transmission. In the case that the localized collaboration range $R_{C}$ is less than or equals to the one-hop communication distance (as in our simulations), the adjacent nodes can directly exchange information and thus no data routing is needed. In the case that $R_{C}$ is greater than the one-hop communication distance but less than $m(m \geqslant 2)$ times the one-hop communication distance, data routing will be needed but the data transmission will be finished within at most $m$ steps. Because node wakeup is coupled with data transmission, it will be an interesting yet open problem to design a cost-effective data routing scheme for the proposed biologically-inspired SWC method. 


\section{REFERENCES}

[1] I. F. Akyildiz, W. Su, Y. Sankarsubramaniam, and E. Cayirci, "Wireless sensor networks: A survey," Comput. Netw., vol. 38, no.4, pp. 393-422, Mar. 2002.

[2] W. Zhang, "A Probabilistic Approach to Tracking Moving Targets with Distributed Sensors", IEEE Trans. Sys., Man, Cyb.-Part A, vol. 37, no. 5, pp721-731, 2007.

[3] A. Sayeed, D. Estrin, G. Pottie, and K. Ramchandran, Eds., "Special issue on self-organizing distributed collaborative sensor networks," IEEE J. Sel. Areas Commun., vol. 23, no. 4, pp. 689-872, Apr. 2005.

[4] D. Li, K. D. Wong, Y. H. Hu, and A. M. Sayeed, "Detection, classification, and tracking of targets," IEEE Signal Process. Mag., vol. 19, no. 3, pp. 17-29, Mar. 2002.

[5] F. Zhao, J. Shin, and J. Reich, "Information-driven dynamic sensor collaboration," IEEE Signal Process. Mag. vol 19, pp. 61 - 72, March 2002.

[6] S. Kumar, F. Zhao, and D. Shepherd., "Collaborative signal and information processing in microsensor networks," IEEE Signal Process. Mag., vol. 19, no. 2, pp13-14, Mar. 2002.

[7] S. Meguerdichian, F. Koushanfar, M. Potkonjak, and M.B. Srivastava, "Coverage Problems in Wireless Ad-Hoc Sensor Networks," IEEE Infocom, pp 1380-1387, April 2001.

[8] G. Chao, and P. Mohapatra, "Power Conservation and Quality of Surveillance in Target Tracking Sensor Networks", MobiHoc, pp. 129-143, May 2004.

[9] S. Pattem, S. Poduri, and B. Krishnamachari, "Energy-Quality Tradeoffs for Target Tracking in Wireless Sensor Networks", $2^{\text {nd }}$ International Workshop on Information Processing in Sensor Networks, pp.32-46. April 2003.

[10] W. Zhang and G. Cao. "DCTC: Dynamic Convoy Tree-Based Collaboration for Target Tracking in Sensor Networks", IEEE Trans. Wirel. Commun., vol. 3, no. 5, pp.1689-1701, 2004.

[11]W. Ye, J. Heidemann, and D. Estrin, "An energy-efficient MAC protocol for wireless sensor networks," INFOCOM, pp. 1567 - 1576, June 2002.

[12]F. Ye, G. Zhong, J. Cheng, S.W. Lu, and L.X. Zhang, "Peas: a robust energy conserving protocol for long-lived sensor networks", IEEE International Conference on Network Protocols, pp200-201, Nov. 2002.

[13] A. Cerpa and D. Estrin, “ASCENT: Adaptive Self-Configuring Sensor Networks Topologies”, INFOCOM 2002.

[14]B. Chen, K. Jamieson, H. Balakrishnan, and R. Morris, "Span: an Energy-Efficient Coordination Algorithm for Topology Maintenance in Ad Hoc Wireless Network", ACM Wirel. Netw. J., vol.8, no.5, pp.481-494, Sep. 2002.

[15]C. Schurgers, V. Tsiatsis, and M. Srivastava, "STEM: Topology management for energy efficient sensor networks," IEEE International Conference on Aerospace, pp78-89, Mar. 2002

[16] Ian A. Gravagne and Robert J. Marks, "Emergent Behaviors of Protector, Refugee, and Aggressor Swarms", IEEE Trans. Sys., Man, Cyb.-Part B, vol. 37, no. 2, pp471-476, 2007.

[17] E. Bonabeau, M. Dorigo, and G. Theraulaz, Swarm Intelligence: from Natural to Artificial Systems, Oxford University Press, 1999.

[18] M. Dorigo and T. Stützle, Ant Colony Optimization. Cambridge, MA: MIT Press, 2004.

[19] M. Dorigo, V. Maniezzo, and A. Colorni, "Ant system: Optimization by a colony of cooperating agents," IEEE Trans. Syst., Man, Cybern. B, vol. 26, no. 1, pp. 29-41, Feb. 1996.

[20] T. Stützle , H. H. Hoos, Max-Min Ant system, Future Gener. Comp. Sys., vol.16 no.9, pp.889-914, June 2000.

[21]K. Verbeeck, A. Nowé, "Colonies of Learning Automata”, IEEE Trans. Syst., Man, Cybern. B, vol. 32, no. 6, pp. 772 - 780, Dec. 2002.

[22] K. H. Low, W.K. Leow, M.H., Jr., "Autonomic mobile sensor network with self-coordinated task allocation and execution”, IEEE Trans. Sys., Man, Cyb._Part C, vol. 36, no. 3, pp315 - 327, 2006.

[23] S. S. Iyengar, H.-C.Wu, N. Balakrishnan, S. Y.Chang, "Biologically Inspired Cooperative Routing for Wireless Mobile Sensor Networks”, IEEE Systems Journal, vol.1 no.1, pp29 - 37, 2007.

[24] P. Boonma, P. Champrasert and J. Suzuki, "BiSNET: A Biologically-Inspired Architecture for Wireless Sensor Networks", The Second IEEE International Conference an Automatic and Autonomous Systems, pp.54, Silicon Valley, California, USA, July 2006.

[25] M. Britton, V. Shum, L. Sacks and H. Haddadi, "A biologically inspired approach to designing wireless sensor networks", The Second European Workshop on Wireless Sensor Networks, pp.256-266, 2005.

[26] Werner-Allen G. et al, "Firefly-Inspired Sensor Network Synchronicity with Realistic Radio Effects", Proceedings of ACM SenSys'05, pp142-153, 2005.

[27] L. Miao, H. Qi, F. Wang, "Biologically-inspired self-deployable heterogeneous mobile sensor networks", IEEE/RSJ International Conference on Intelligent Robots and Systems (IROS), pp2363 - 2368, Aug. 2005

[28] A. Boukerche, X. Fei, R. B. Araujo, "An energy aware coverage-preserving scheme for wireless sensor networks", in Proceedings of the 2nd ACM international workshop on Performance evaluation of wireless ad hoc, sensor, and ubiquitous networks (PE-WASUN '05), pp205 - 213, Aug. 2005.

[29] R. Wehner, M. V. Srinivasan, "Searching behaviour of desert ants", Journal of Comparative Physiology A: Neuroethology, Sensory, Neural, and Behavioral Physiology, Vol.42, No. 3, pp315-338, 1981. 
[30]R. Wehner, , K. Gallizzi, C. Frei, M. Vesely, "Calibration processes in desert ant navigation: vector courses and systematic search", Journal of Comparative Physiology A: Neuroethology, Sensory, Neural, and Behavioral Physiology, Vol.188, No. 9, 683-693, 2002

[31] T. Merkle, M. Knaden, R. Wehner, "Uncertainty about nest position influences systematic search strategies in desert ants ", Journal of Experimental Biology, Vol.209, 3545-3549,2006

[32] M. V. Srinivasan, "Animal navigation: Ants match as they march", Nature, Vol. 392, 660-661, 1998.

[33]X.Yuan, S.X.Yang, "Virtual Assembly With Biologically Inspired Intelligence", IEEE Trans. Sys., Man, Cyb.-Part C, vol. 33, no. 2, pp159-167, 2003.

[34] L.Wang, C. Singh, "Reliability-Constrained Optimum Placement of Reclosers and Distributed Generators in Distribution Networks Using an Ant Colony System Algorithm”, IEEE Trans. Sys., Man, Cyb._Part C, vol. 38, no. 6, pp757 - 764, 2008.

[35] W.Chen, J. Zhang, “An Ant Colony Optimization Approach to a Grid Workflow Scheduling Problem with Various QoS Requirements”, IEEE Trans. Sys., Man, Cyb._Part C, vol. 39, no. 1, pp29 - 43, 2009.

[36] K.Konstantinidis, G.C.Sirakoulis, I.Andreadis, "Design and Implementation of a Fuzzy-Modified Ant Colony Hardware Structure for Image Retrieval”, IEEE Trans. Sys., Man, Cyb._Part C, vol. 38, no. 6, pp520 -533, 2009.

\section{APPENDIX A. DEFINITIONS FOR ThEOREMS 1 AND 2}

Throughout the paper, we use $\mathrm{P}\{\}$ and $\mathrm{P}\{\mid\}$ to represent prior probability and conditional probability, respectively. The indicator of food existence confirmation triggered by a true target is defined as

$$
T_{j}(k)=\left\{\begin{array}{lc}
1 & \begin{array}{l}
\text { The ture target triggers } A_{j} \text { 's confirmation } \\
\text { of target existence in the } k \text {-th Round. }
\end{array} \\
0 & \text { otherwise }
\end{array}\right.
$$

and the indicator of food existence confirmation triggered by clutters is defined as

$$
C_{j}(k)=\left\{\begin{array}{lc}
1 & \begin{array}{l}
\text { The clutters triggers } A_{j} \text { 's confirmation } \\
\text { of target existence in the } k \text {-th Round. }
\end{array} \\
0 & \text { otherwise }
\end{array}\right.
$$

From (2.3), (A.1) and (A.2), we can represent the indicator of food existence confirmation as

$$
D_{j}(k)=\left\{\begin{array}{cc}
1 & T_{j}(k)=1 \\
1 & T_{j}(k)=0 \text { and } C_{j}(k)=1 \\
0 & T_{j}(k)=0 \text { and } C_{j}(k)=0
\end{array}\right.
$$

One important parameter of the sensor node is the detection probability, $P_{D}$. It represents the probability that one ant confirms "Target Existence" if it is awake and the target is in its sensing range:

$$
P_{D}=P\left\{T_{j}(k)=1 \mid S_{j}(k)=1, E_{j}(k)=1\right\}
$$

Obviously, it is impossible that the target triggers $A_{j}$ 's confirmation of target existence in the case that the ant does not search or the target does not appear in its sensing range. We have

$$
P\left\{T_{j}(k)=1 \mid S_{j}(k)+E_{j}(k) \leq 1\right\}=0
$$

Another important parameter of the sensor is the probability of false alarm, $P_{f}$. It represents the 
probability that one ant reports "Target Existence" caused by clutters:

$$
P_{f}=P\left\{C_{j}(k)=1 \mid S_{j}(k)=1, T_{j}(k)=0\right\}
$$

Obviously, it is impossible that the clutters trigger $A_{j}$ 's confirmation of target existence in the case that the sensor is asleep or the target triggers $A_{j}$ 's confirmation. We have

$$
P\left\{C_{j}(k)=1 \mid S_{j}(k)-T_{j}(k) \leq 0\right\}=0
$$

\section{APPENDIX B. PROOF OF THEOREM 1}

\section{B.1. Determining the upper bound of $|\operatorname{vicinity}(j)|$}

Consider that the target location follows the uniform distribution within the surveillance zone. It represents the least apriori information about the target location, and hence is the most difficult case to deal with. The probability that the target appears in the sensing range of a sensor node, $P_{E}$, is calculated as

$$
P_{E}=\pi s^{2} /\|\Omega\|
$$

The deployment of sensor nodes in a given field can be modeled as a realization of a spatial point process, governing the event that $n$ nodes are located in a bounded area. When $n$ is large and the nodes are distributed independently and uniformly, the number of sensor nodes in an arbitrary area inside the surveillance zone obeys the homogeneous Poisson Process. That is to say, the number of nodes in any subset $\Theta$ of the surveillance zone $\Omega$ obeys Poisson distribution with mean $n\|\Theta\| /\|\Omega\|$, where $\|\Theta\|$ and $\|\Omega\|$ are the areas of $\Theta$ and $\Omega$, respectively. Therefore we obtain the probability distribution function of the node number in $\operatorname{vicinity}(j)$ as

$$
\operatorname{pmf}_{1}(M) \triangleq P\{|\operatorname{vicinity}(j)| \leq M\}=\sum_{r=0}^{r=M} e^{-\lambda_{1}} \lambda_{1}^{r} / r !
$$

where $\lambda_{1}=n \pi R_{c}^{2} /\|\Omega\|$. Given the confidence degree $\beta$, we can determine $M_{\max }$, the upper bound of the node number in vicinity $(j)$, satisfying

$$
\operatorname{pmf}_{1}\left(M_{\max }-1\right)<\beta \leq p m f_{1}\left(M_{\max }\right)
$$

\section{B.2. Determining $P\left\{D_{j}(k)=1\right\}$}

$$
\begin{aligned}
& P\left\{D_{j}(k)=1\right\}=P\left\{D_{j}(k)=1, S_{j}(k)=1\right\}+P\left\{D_{j}(k)=1, S_{j}(k)=0\right\} \\
& =P\left\{D_{j}(k)=1, S_{j}(k)=1\right\}
\end{aligned}
$$


(Because the sleep node can not detect the possible target and thus no confirmation will be reported.)

$=P\left\{T_{j}(k)=1, S_{j}(k)=1\right\}+P\left\{T_{j}(k)=0, C_{j}(k)=1, S_{j}(k)=1\right\}$

(Using (A.3))

$=P\left\{T_{j}(k)=1, S_{j}(k)=1, E_{j}(k)=1\right\}+P\left\{T_{j}(k)=1, S_{j}(k)=1, E_{j}(k)=0\right\}+P\left\{T_{j}(k)=0, C_{j}(k)=1, S_{j}(k)=1\right\}$

$=P\left\{T_{j}(k)=1, S_{j}(k)=1, E_{j}(k)=1\right\}+P\left\{T_{j}(k)=0, C_{j}(k)=1, S_{j}(k)=1\right\}$

(Because $P\left\{T_{j}(k)=1, S_{j}(k)=1, E_{j}(k)=0\right\}=0$, i.e. it is impossible to obtain $T_{j}(k)=1$ in the absence of target.)

$\begin{aligned}= & P\left\{S_{j}(k)=1\right\} P\left\{E_{j}(k)=1 \mid S_{j}(k)=1\right\} P\left\{T_{j}(k)=1 \mid S_{j}(k)=1, E_{j}(k)=1\right\} \\ & +P\left\{S_{j}(k)=1\right\} P\left\{T_{j}(k)=0 \mid S_{j}(k)=1\right\} P\left\{C_{j}(k)=1 \mid S_{j}(k)=1, T_{j}(k)=0\right\} \\ = & P\left\{S_{j}(k)=1\right\} P\left\{E_{j}(k)=1\right\} P\left\{T_{j}(k)=1 \mid S_{j}(k)=1, E_{j}(k)=1\right\} \\ & +P\left\{S_{j}(k)=1\right\} P\left\{T_{j}(k)=0 \mid S_{j}(k)=1\right\} P\left\{C_{j}(k)=1 \mid S_{j}(k)=1, T_{j}(k)=0\right\}\end{aligned}$

(In surveillance, the value of $E_{j}(k)$ is unknown before the sleep/wakeup design, thus the design of $S_{j}(k)$ is independent of $E_{j}(k)$.)

$=w_{j}(k) P_{E} P_{D}+w_{j}(k) P\left\{T_{j}(k)=0 \mid S_{j}(k)=1\right\} P_{f}$

$=w_{j}(k) P_{E} P_{D}+w_{j}(k)\left(P\left\{T_{j}(k)=0, E_{j}(k)=1 \mid S_{j}(k)=1\right\}+P\left\{T_{j}(k)=0, E_{j}(k)=0 \mid S_{j}(k)=1\right\}\right) P_{f}$

$=w_{j}(k) P_{E} P_{D}+w_{j}(k)\left(P\left\{T_{j}(k)=0, E_{j}(k)=1 \mid S_{j}(k)=1\right\}+P\left\{E_{j}(k)=0 \mid S_{j}(k)=1\right\}\right) P_{f}$

(Using the fact that $E_{j}(k)=0$ results in $T_{j}(k)=0$. .)

$=w_{j}(k) P_{E} P_{D}+w_{j}(k)\left(P\left\{T_{j}(k)=0, E_{j}(k)=1 \mid S_{j}(k)=1\right\}+P\left\{E_{j}(k)=0\right\}\right) P_{f}$

(Because $S_{j}(k)$ is independent of $E_{j}(k)$ as mentioned above.)

$=w_{j}(k) P_{E} P_{D}+w_{j}(k)\left(P\left\{E_{j}(k)=1 \mid S_{j}(k)=1\right\} P\left\{T_{j}(k)=0 \mid E_{j}(k)=1, S_{j}(k)=1\right\}+1-P_{E}\right) P_{f}$

$=w_{j}(k) P_{E} P_{D}+w_{j}(k)\left(P\left\{E_{j}(k)=1\right\}\left(1-P_{D}\right)+1-P_{E}\right) P_{f}$

$=w_{j}(k) P_{E} P_{D}+w_{j}(k)\left(1-P_{E} P_{D}\right) P_{f}$

$=w_{j}(k)\left(P_{f}+P_{E} P_{D}\left(1-P_{f}\right)\right)$

\section{B.3. Determining the maximum Pheromone Threshold}

The new pheromone increment in (2.6) is rewritten as

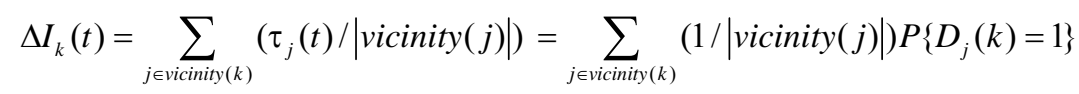

Put (B.4) into (B.5), we have

$\left.\Delta I_{k}(t)=\sum_{j \in v i c i n i t y(k)}(1 / \mid \operatorname{vicinity}(j))\right) w_{j}(k)\left(P_{f}+P_{E} P_{D}\left(1-P_{f}\right)\right)$

and further make decision with the confidence degree $\beta$ that

$$
\begin{aligned}
\Delta I_{k}(t) & \leq M_{\text {max }} \cdot(1 /|\operatorname{vicinity}(j)|) w_{j}(k)\left(P_{f}+P_{E} P_{D}\left(1-P_{f}\right)\right) & & (\text { Using }(\text { B. } 3)) \\
& \leq M_{\text {max }}\left(P_{f}+P_{E} P_{D}\left(1-P_{f}\right)\right) /|\operatorname{vicinity}(j)| \underline{\underline{\Delta}} H & & \text { (Because } \left.w_{j}(k) \leq 1\right)
\end{aligned}
$$


The ultimate pheromone accumulation in (2.7) is

$I_{j}(t+1)=\min \left\{I_{j}^{\max }, \max \left\{I_{j}^{\min },(1-\rho) I_{j}(t)+\Delta I_{j}(t)\right\}\right\} \leq \min \left\{I_{j}^{\max }, \max \left\{\begin{array}{l}\min \\ j\end{array},(1-\rho) I_{j}^{\max }+H\right\}\right\}$

Therefore we can choose $I_{j}^{\max }$ as

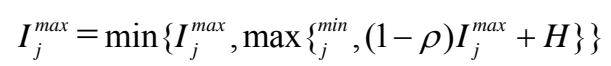

$\Rightarrow I_{j}^{\max }=(1-\rho) I_{j}^{\max }+H$

$\Rightarrow I_{j}^{\max }=H / \rho=M_{\max }\left(P_{f}+P_{E} P_{D}\left(1-P_{f}\right)\right) /(\rho|\operatorname{vicinity}(j)|)$

Put (B.1) into (B.6), we have (3.5).

End of proof.

\section{ApPendix C. Proof of TheOREM 2}

\section{C.1. Determining the surveillance probability}

The surveillance probability, $P_{g}(k)$, is

$P_{g}(k) \triangleq P\{$ the target is confirmed by $\Psi(k)$ in the $k$-th round $\}$

$=1-P$ the target is not confirmed by $\Psi(k)$ in the $k$-th round $\}$

$=1-\prod_{A_{j} \in \Psi(k)} P\left\{D_{j}(k)=0 \mid E_{j}(k)=1\right\}$

$=1-\prod_{A_{j} \in \Psi(k)}\left(1-P\left\{D_{j}(k)=1 \mid E_{j}(k)=1\right\}\right)$

$=1-\prod_{A_{j} \in \Psi(k)}\left(1-P\left\{T_{j}(k)=1, S_{j}(k)=1 \mid E_{j}(k)=1\right\}-P\left\{T_{j}(k)=0, C_{j}(k)=1, S_{j}(k)=1 \mid E_{j}(k)=1\right\}\right)$

Now we need to determine the two probabilities in the right side of (C.1). The first probability can be derived as follows

$P\left\{T_{j}(k)=1, S_{j}(k)=1 \mid E_{j}(k)=1\right\}=P\left\{S_{j}(k)=1 \mid E_{j}(k)=1\right\} P\left\{T_{j}(k)=1 \mid E_{j}(k)=1, S_{j}(k)=1\right\}$

(In surveillance, the value of $E_{j}(k)$ is unknown before the sleep/wakeup design, thus the design of $S_{j}(k)$ is independent of $E_{j}(k)$.)

$=P\left\{S_{j}(k)=1\right\} P\left\{T_{j}(k)=1 \mid S_{j}(k)=1, E_{j}(k)=1\right\}=w_{j}(k) P_{D}$

The second probability can be obtained as follows

$$
\begin{aligned}
& P\left\{T_{j}(k)=0, C_{j}(k)=1, S_{j}(k)=1 \mid E_{j}(k)=1\right\} \\
& =P\left\{T_{j}(k)=0, C_{j}(k)=1 \mid E_{j}(k)=1, S_{j}(k)=1\right\} P\left\{S_{j}(k)=1 \mid E_{j}(k)=1\right\} \\
& =P\left\{S_{j}(k)=1\right\} P\left\{T_{j}(k)=0, C_{j}(k)=1 \mid E_{j}(k)=1, S_{j}(k)=1\right\} \\
& =P\left\{S_{j}(k)=1\right\} P\left\{T_{j}(k)=0 \mid E_{j}(k)=1, S_{j}(k)=1\right\} P\left\{C_{j}(k)=1 \mid E_{j}(k)=1, S_{j}(k)=1, T_{j}(k)=0\right\} \\
& =P\left\{S_{j}(k)=1\right\} P\left\{T_{j}(k)=0 \mid E_{j}(k)=1, S_{j}(k)=1\right\} P\left\{C_{j}(k)=1 \mid S_{j}(k)=1, T_{j}(k)=0\right\}
\end{aligned}
$$


(Because $C_{j}(k)$ has nothing to do with $E_{j}(k)$ in the case that $T_{j}(k)$ is known.)

$=w_{j}(k)\left(1-P_{D}\right) P_{f}$

Put(C.2) and (C.3) into (C.1), we have

$$
P_{g}(k)=1-\prod_{A_{j} \in \Psi(k)}\left(1-w_{j}(k) P_{D}-w_{j}(k)\left(1-P_{D}\right) P_{f}\right)=1-\left(1-w_{j}(k)\left(P_{D}+\left(1-P_{D}\right) P_{f}\right)\right)^{|\Psi(k)|}
$$

\section{C.2. Determining the lower bound of $|\Psi(t)|$}

Due to the random node deployment and random target movement, $|\Psi(k)|$ is a random variable. Therefore (C.4) can not be calculated directly. Here we derive, with a certain confidence degree, the low bound of $|\Psi(k)|$ to support the further derivation of the minimum wakeup probability.

The deployment of sensor nodes in a given field can be modeled as a realization of a spatial point process, governing the event that $n$ nodes are located in a bounded area. When $n$ is large and the nodes are distributed independently and uniformly, the number of sensor nodes in an arbitrary area inside the surveillance zone obeys the homogeneous Poisson Process. A property of this process is that the number of nodes in any subset $\Theta$ of the surveillance zone $\Omega$ obeys Poisson distribution with mean being $n\|\Theta\| /\|\Omega\|$, where $\|\Theta\|$ and $\|\Omega\|$ are the areas of $\Theta$ and $\Omega$, respectively. We obtain the probability distribution function of the node number in $R(k)$ as

$$
\operatorname{pmf}_{2}(M) \stackrel{\Delta}{=} P\{|\Psi(t)| \leq M\}=\sum_{r=0}^{r=M} \frac{e^{-\lambda_{2}} \lambda_{2}^{r}}{r !}
$$

where $\lambda_{2}=n \pi s^{2} /\|\Omega\|$ and $s$ is the sensing range of nodes. Given the confidence degree $1-\alpha$, we can determine $M_{\min }$, the lower bound of $|\Psi(t)|$, satisfying

$$
\operatorname{pmf}_{2}\left(M_{\min }\right) \leq \alpha<p m f_{2}\left(M_{\min }+1\right)
$$

\section{C.3. Determining the minimum wakeup probability}

After determining the lower bound of $|\Psi(t)|$ using (C.5) and (C.6), $P_{g}(t)$ in (C.4) can be derived with the confidence degree $1-\alpha$

$$
P_{g}(k)=1-\left(1-w_{j}(k)\left(P_{D}+\left(1-P_{D}\right) P_{f}\right)\right)^{|\Psi(k)|}
$$

Let $w_{0}(k)=\min \left\{w_{j}(k) \mid j \in \Psi(k)\right\}$, we have 
$P_{g}(k) \geq 1-\left(1-w_{0}(k)\left(P_{D}+\left(1-P_{D}\right) P_{f}\right)\right)^{|\Psi(k)|} \geq 1-\left(1-w_{0}(k)\left(P_{D}+\left(1-P_{D}\right) P_{f}\right)\right)^{M_{\min }}$

(With the confidence degree $1-\alpha$ )

Given the minimum surveillance probability, $P_{\min }$, we obtain the minimum node deployment with the confidence degree $1-\alpha$ by

$$
\begin{aligned}
& 1-\left(1-w_{0}(k)\left(P_{D}+\left(1-P_{D}\right) P_{f}\right)\right)^{M_{\min }} \geq P_{\min } \Leftrightarrow 1-P_{\min } \geq\left(1-w_{0}(k)\left(P_{D}+\left(1-P_{D}\right) P_{f}\right)\right)^{M_{\min }} \\
& \Leftrightarrow w_{0} \geq\left(1-\left(1-P_{\min }\right)^{\frac{1}{M_{\min }}}\right) /\left(P_{D}+\left(1-P_{D}\right) P_{f}\right)
\end{aligned}
$$

Thus we obtain the minimum wakeup probability $w_{\min }$ as

$$
w_{\min }=\left(1-\left(1-P_{\min }\right)^{\frac{1}{M_{\min }}}\right) /\left(P_{D}+\left(1-P_{D}\right) P_{f}\right)
$$

Substituting $I_{j}(k+1)$ in (2.8) by $I_{j}^{\min }, w_{j}(k+1)$ in (2.8) by $w_{\min }$ in (C.8), and putting (B.6) into (2.8), we can obtain the minimum pheromone, $I_{j}^{\min }$, as

$$
I_{j}^{\min }=\frac{M_{\max }\left(P_{f}+P_{E} P_{D}\left(1-P_{f}\right)\right)\left(1-\left(1-P_{\min }\right)^{\frac{1}{M_{\min }}}\right)}{\rho|\operatorname{vicinity}(j)|\left(P_{D}+\left(1-P_{D}\right) P_{f}\right)}
$$

Put (B.1) into (C.9), we have (3.10).

End of proof. 